\title{
TOWARDS HUMAN ORIENTED WWW
}

\author{
Alex Abramovich \\ Hazionut 38/13, Haifa, 35312,Israel,10 Devikarumariamman second street, vijayanagar, \\ velachery, chennai 600042
}

\begin{abstract}
The ultimate aim of computer science is to assist humans with reasoning, decision-making and chores for their profession/living everyday complex problem solving. In other words, the target state of human-machine symbiosis is characterized by using a machine as a personal intellectual assistant. A principal impediment consists in the multi-disciplinary nature of the profession/living human activity. A customer, as a rule, is not a specialist in all related domains. This implies that an achievement of the posed problem is a providing a personal intellectual assistant with the multidisciplinary knowledge. This paper deals with an approach to the problem via Web extension that contains Total Human Experience (THE Web).
\end{abstract}

Key words: Human experience, ontology, activity proposition, personal world, domain world

\section{INTRODUCTION}

Without regard to the biochemical ground of human brain functioning note that human reasoning accompanies the physical/mental human activities and is an activity itself. Such as it provides an achievement of current activity as well as operates on the set of related activities and activities' states. In this connection human has to be aware of both his current activity and all related/influenced activities. Thereby he must operate of activities' knowledge.

Reasoning as human activity is executed in the following modes by a generation of executive directives or/and by calling the service providers: 
- Recognition of an activity initialization's necessity (that is, a latchup of an activity as a component of cause-and-effect relations)

- Recognition of an activity initialization's availability (that is, a latchup of constituents of an activity initialization's conditions)

- An acquisition of missing data/knowledge including also a probability of the problems solution generation on basis of corresponding cause-andeffect relations

- A maintenance of an activity steps' execution

- A maintenance of an activity living conditions' protection. This implies an activity related KR standpoint, namely,

- It exists a knowledge, that is a system of socio/physical/mental causal relationships' representation, where every node is causality in a M.Bunge definition [15] (that is, a permanent and mono-semantic production) represented by generalized executive plan (Activity Proposition (see below))

- Semantics of causality as well as an activity component's is function of its position in causalities system or in Activity Proposition correspondingly

- Meaning of knowledge component is a representation of goal suitable available resource.

The present paper is based on the above-mentioned standpoint.

We will consider only verbalized experience. Verbalization practice, undoubtedly, is one of the most ancient human activities.

Multi-millennial endeavor of thought expression brought to beginnings of different languages under different grammars that, in turn, determine how the conceptual structures are linearized as strings of words in a sentence. In spite of all differences it exist 3 approach of the linearization [9], namely, SVO (English and Chinese, for example), VSO (Irish and Biblical Hebrew, for example) and SOV (Latin and Japanese, for example), where $\mathrm{S}$ is a subject, $\mathrm{V}$ is a verb, and $\mathrm{O}$ is an object.

Judging from this, the basis human word usage's trend is an activity representation. Generally speaking, an activity is a corresponding experience's knowledge utilization. Activities' execution is an origin of problems. Problems' solving generates a novel knowledge. A multifold physical or mental (both successful and failed) execution of activities constitutes a Total Human Experience (THE).

Thus, we dispose, at least, of one a proven THE representation approach (that is, a verbal approach). Note that THE is represented also by various materialized forms.

We hold an interest in Web representation of THE (THE Web).

It is naturally to assume that Web KR inherits from the existing forms of an experience representation. However, we don't see it in practice. Existing 
Web knowledge-, ontology-, semantics-, reasoning-, decision-makingrepresentation approaches are not related

directly with a verbal practice of activities representation. Existing Web $\mathrm{KR}$ approaches utilize the formal methods and languages that grounded by various world models, generative interoperability/reuse issues.

In lieu of formal methods THE Web applies a semantic marking of natural language sentences.

\section{ACTIVITY PROPOSITION}

Note that man-Web symbiosis' interface aimed to both target setting in form of queries, requirements, situations' declarations, goal declarations as well as answers in form of quantitative/qualitative information. Thereby, the interface's lexicon is narrowed by framework of Web visitor's activities. To Sowa "The lexicon is the bridge between a language and the knowledge expressed in that language" [9]. We will take liberties with this formula. The lexicon is the bridge between knowledge, expressed in the language, and the activity execution. Lexicon consists of words and idioms. The words represent subjects, verbs, objects, auxiliary words, pronouns, interrogative words, etc. Part of lexicon is easy recognizable basic vocabulary (semantically lucid). A residuary (major) part depends on the context. We take an interest in the second part that represents a difficulty for the standard parsers.

Standard parsers are not context specified.

How is it possible an orienting of parser to the current context?

The current context's awareness gives rise to the binding words with meanings. Since semantics is, in general, the study of the relationship between words and meanings [10], the current context is utilized as input of the semantic analysis. The current context of communication is specified by the operating activities. So the target settings' as well as the answers' lexicon consists of activities related vocabulary (a contextual lexicon). As activities belong to the certain domains, the contextual lexicon belongs to the domain ontology.

On condition that, an intercourse's participants are the operating activities aware, it is possible to adequately interpret and to respond to input/output text.

Therefore THE Web intercourse must be anticipated by an agreement on all operating or desirable set of activities as well as about accompanying lexicon (that is, professional/living ontology). 


\section{AN AGREEMENT OF ACTIVITIES}

Granting requirement that THE Web must be available for everybody, it is necessary to keep in mind the following key features of system design by Everyman [11]:

- There is no a priori personal THE Web service to be built.

- Everyman ("end-user" who is not computer expert), as a rule, agrees at the most than to alter associations among participating components in the dynamic conditions and/or else to intercommunicate on the natural language.

- "The large majority of users cannot-and do not want to-be engaged in any kind of "programming" other than simple scripting. However, they are able to assemble components together by "wiring" them. This means that components should provide encapsulated chunks of behavior in the form of "probes" for structural coupling with other components, and the supporting environment should provide the means for "translating" the structural inter-component relationships to overall microworld behavior [12,13]". THE Web agreement on activities is based on foregoing features, and lies in

- an assemblage by user and a fitting of separate actions for achievement of personal objectives

- an updating by user the existing service providing architectures by removing and/or insertion suitable functional components that are represented according to text or by sight

- a target setting by user in the form of what is given and of what is need or in the form of what is given only or in the form of what is need only

- an activity description by user as a context marking (in the network of a facilitating interface)

During an activity structure agreement THE Web engine must

- to submit a basis activity ontology to user approval (in that way THE Web engine learns user lexicon)

- to generate an activity description as a semantic marking sentence in IO-SV-TO form, where IO-SV-TO is an activity's Initial Objects (as a cause's conditions), Subject and Verbs (as a cause) and Target Objects (as an effect) accordingly

- to generate an activity's semantic assurance network, inclusive of cause-and-effect relations that join an activity's components. 


\section{CONTEXT MARKING}

Context marking of an activity's description sentence fixes the contribution of an activity's components to the activity's goals achievement. The marking is distributed into three groups, namely,

- Objectives

- Subject

- Verbs.

THE Web allows denominating

- Objectives of place conditions, of time conditions, of facts presence, of actions presence, of goal conditions and other conditions under titles "On condition that" and "With the view of".

- Subjects under titles "Main actor" and "Execute"

- Verbs under titles "Execute" and "programme".

Current activity determination starts from a correlation the basis generic domain activity's proposition with private needs.

Example 1. Generic Activity proposition of building construction

On condition that:

Where : place of the building according to the project documentation

When : period : contract period

by direction of : project documentation

With the view of : an achievement of a design statement

Main actor: organization : Building Contractor

Execute : building construction :

Programme :

It's given : project documentation

Do :

I(foundation, water-supply, power supply, sewerage system),

For 1 to the "number of floors according to the project documentation" by step 1

Do : floor, ceilingl,

Do : (scavenging, finishing, planting of greenery),

where "[]" holds a sequence of activities, and "()" holds activities that are executed at the same time.

First of all it inserts a name of Building Contractor and data from project documentation. After that it is necessary to correlate all included activities, namely, foundation building, water-supply building, power supply wiring, etc. A word's record under different context marks involves it different utilizations. Since a usage's context is determinate by an activity proposition, the chief task is a detection of operating activity. Discovered context launches the new perspectives in the

Nature Language Understanding area. 


\section{TOWARDS A NATURAL LANGUAGE UNDERSTANDING BY MEANS OF CONTEXT MARKING}

An orientation of European Commission and other interested parties to the Web personalized computing service for Everyman sets with a new force a problem of Natural Language Understanding (NLU).

Typographical method of text marking was served as conceptual model for Web mark-up languages' developers. But human intercourse doesn't reduce to an exchange of documents. Therefore existing mark-up languages are rough tools for maintenance of Web-user nature language interface. According to M. Polanyi [1], the components of an optimally organized system must not be further partible in the certain, defined for this system, ratio.

M. Polanyi made out of a system's components at a ratio of their contributions to the goal achievement. A component's position in the system's organization defines its semantics.

Its contribution defines a meaning of the component. NLU lies in the recognition of semantics and meaning of propositions.

Following M. Polanyi, it is necessary to be aware of interlocutors' goals or a reader's and text's goals or user's and software's goals.

This implies that NLU is characterized by a presence of two parties, and we must be acquainted with both parties of the NLU process.

In the focus of our attention is an establishment of an interaction between Web user and THE via AmI [2,3] technology .

"The concept of Ambient Intelligence (AmI) provides a vision of the Information Society where the emphasis is on greater userfriendliness, more efficient services support, user-empowerment, and support for human interactions. People are surrounded by intelligent intuitive interfaces that are embedded in all kinds of objects and an environment that is capable of recognizing and

responding to the presence of different individuals in a seamless, unobtrusive and often invisible way." Taken from the ISTAG scenarios document (see ftp://ftp.cordis.lu/pub/ist/docs/istagscenarios2010.pdf)

AmI-engine presupposes a presence of following features:

- A mobility and an ubiquity;

- An autonomic computing;

- An availability for Everyman;

- A personalization.

Realization of these features is possible on conditions that AmI-engine is aware of AmI's owner activities' models including these 
activities' domains of existence. At that a personalization for Everyman implies an intercourse on the natural language as well as

an autonomic computing implies a necessity of self-maintained information acquisition including from texts.

Thus, AmI-engine causes NLU parties awareness that grounds an opportunity of NLU carrying out. At that an opposition of two extremes on the semantics-based spectrum (that is, R. Montague's formalism and R. Schank's lack of a precise formalism [9]) is solved via Activity Propositions by a strict presentation of word usage context, on the one hand, and

by multiplicity of such presentations, on the other hand.

\subsection{Semantics of Activity Proposition}

From an infinite set of eventual word usage contexts it is necessary to find contexts that defined the generic words' semantics. Such contexts are both physical and mental generic activities' descriptions (activities propositions), where a generic activity is domain's constitutive activity.

A strict of generic activity proposition is caused by its an optimal organization that is verified by scientific (that is, by means formalisms) and by an experiment way.

Every name's semantics depends on its syntactic position in an operating generic activity's proposition, with inherits syntactic rules of a compound sentence.

Could say that Activity Proposition represents a semantic (in Polanyi sense) name space's sharing (Activity's Ontology (AO)).

For every $\mathrm{AO}$ it is fixed the name list that represents an activity's foundation_architecture. A presence of this, so called,

MinContext indicates an occurrence of a corresponding activity.

Word semantics' understanding comes to recognition in the ambient name space a MinContext of an operating domain generic activity's application (OPERA).

\subsection{MinContexts' relatedness}

Since a main problem consists in an acquisition of operating activities, MinContext's recognition is a simple FOL based procedure. It is possible utilizing of MinContext procedure both together with syntactic/morphological analysis and as detached mode. As result of the detached mode will be a resume about the text's semantic activity related content. 
MinContext procedure, applied together with syntactic/morphological analysis, will results a semantically verified text translation.

MinContext procedure realizes also a lexical analysis, aimed for an acquisition of activity utilization specificity as well as level of executer proficiency and his personal identification.

OPERA detection implies both its formative activities and related activities, namely:

AmI related (self-controlled) activities and

External related activities.

AmI related activities (by THE Web notation) are the following:

Aexe(name) aimed to the achievement of OPERA's goals;

Repair(name) aimed to the restoration of lost functions and an overcoming of an operational problems as well as to the modernization of an operational structure;

Learning(name) aimed to the acquisition a new constructive knowledge for OPERA life support related;

Protection(name) aimed to protection OPERA from a destructive influence of both internal and external environment;

Provision(name) aimed to the maintenance of the no-break supply of OPERA execution by deliverables as well as by components and data;

Selling(name) aimed to the realization (or to the utilization) of OPERA results;

Direction(name) aimed to an organization of OPERA effective accomplishment,

where name is name of OPERA. This set of internal related activities is derived by AmI-engine from AmI's set of rooted activities (AmI-root). To THE Web notation AmI-root is invariant for customer support outside the dependence on the customer's type (that is, a government, an enterprise, professional and other communities, domain expert or Everyman).

Note that $A m I$ related activities constitute an intelligence environment aimed to maintenance of OPERA's life cycle. THE Web builds an environment one as a multi-agent system (AmI-mas) under FIPA. AmI-mas based sharing of intellectual function together with AP representation standard as well as common AmI-root ontology ground systematization and regulating of FIPA

supported communicative acts [17]. External related activities are activities that input or output is based on the output or input of OPERA correspondingly. In other words, external related activities are activities that relate OPERA by cause-and-effect relations.

Furthermore, every state of the OPERA or OPERA related components, as a rule, are output of any activity that broadens MinContext's family. 
Thereby, MinContexts of OPERA related activities pave an open-ended access to background knowledge (in Shank's sense [6]).

\subsection{MinContext area}

THE engine considers the following text origins:

- Registered user (THE Web service owner, a person or organization, such as a business or an individual as well as provider entity (a person or organization) as well as a requester entity (a person or organization) [according to [16]]),

- Unregistered user as a requester entity [16],

- Software agent (requester agent and provider agent [16]),

- Service provider as provider entity [16],

- E-mail,

- Web document.

Registered user's directives, replies, messages, queries and requirements are considered in a context of AmI run-unit and related activities. In this case MinContext area is known. Unregistered user's directives, replies, messages, queries or requirements are considered after recognition of his problem by means of namespace of AmI-root propositions (AmI ontology) and THE ontologybased browser (O'browser).

O'browser is aimed to the boosted THE Web's look-up. In this case discovered user's goal determines MinContext area.

An activity proposition's executor Aexe initiates software agents as well as service providers. Aexe is one of AmI-engine formative software agents. Its destination is an assembling of a process execution module according to the activity proposition.

With the purpose of the module filling by real meanings, Aexe invokes suitable software agents and service providers. In this case their output must fit the activity proposition context.

E-mail is considered by means of joint for a receiver and a sender activities' MinContexts.

Since Web document reading is initiated by Aexe or by AmI owner in the network of his activity performance, this implies MinContext is known.

Thus AmI-engine always deals with text that semantics belongs to known set of contexts. AmI-engine considers every current mined word as an opening or closing tag, and search a corresponding closing/opening tag in the ambient text space using initiated MinContexts as semantic expectations down to corroboration one of them.

Above-stated reasons indicate that THE NLU can be reduced to relatively simple rules that show what just categories of words may occur on the right 
or the left of a given word, and may be comparable with the Schankian expectations or the cancellation rules of categorial grammar [6].

Generic domain activity propositions causality- and AmI-related activity propositions as well as AP of domain world and AP of personal world (see below) define semantically specified ontology (Onto-surface) that grounded recognition of semantics and meaning of sentences by means utilization the ontology units as opening/closing semantic tags.

In other words, context marking of Activity Propositions (AP marking) represents both marking in the generally accepted sense (that is, by standard set of tags) and dynamic marking by AP owner's tags. Since AP operates with standard and specific sets of actions as well as with logic operations, every named AP component has its own marking, and so AP marking is ndimensional one. Thus AP marking grounds searching of the OPERA and it's addressing.

\section{PERSONAL WORLD}

Personal world (PW) is constituted by set of actual profession/living ( $\mathrm{p} / \mathrm{l})$ activities as well as by knowledge of both personal and borrowed experience (that is, by knowledge of executing earlier $\mathrm{p} / \mathrm{l}$ activities). Every $\mathrm{p} / \mathrm{l}$ activity is correlated with others by time, by place, by power inputs and by cost. Space of correlated $\mathrm{p} / 1$ activities is rank-ordered by AP of personal world (APpw) that

represents a scenario of parallel/sequential executable personal $\mathrm{p} / \mathrm{l}$ activities, which are marked by a special set of tags. THE engine keeps two AP special sets of AmI related semantic tags for both enterprises and natural persons that define an activity's position in the personal world. APpw provides a semantic sharing of personal $\mathrm{p} / 1$ activities as well as of personal $\mathrm{p} / \mathrm{l}$ ontology.

Personal World Activity (PW Activity) represented by APpw is aimed to the achievement of $\mathrm{p} / 1$ goals with cost minimization. A priority of APpw's performance produces a personal causal stipulation of person's behaviors as well as his responses to external occurrences (a personal logic). A corresponding APpw ontology has, therefore, personal semantic features.

A personal logic induces interoperability issue both on the profession and on the living level that must be considered as an operation problem both of PW management and of PWs interaction. In case that a response to an external occurrence is not contradict APpw performance it will meet expectation. If not, a response may be inadequate. In any case THE engine must model

APpw's performance subject to the response. 
THE engine generates APpw on the ground both on information declared by customer and as response to his requirements.

APpw performance is achieved via personal Web portal

- By person as his $\mathrm{p} / \mathrm{l}$ duties

- By person as his response to THE instructions/requirements

- By THE engine's reasonable exploitation of service providers as well as generic and personal activities (THE service).

\section{DOMAIN WORLD}

Domain world is constituted by activities of professional communities, of enterprises and of specialists. THE Web engine keeps AP special sets of AmI related semantic tags that define a profession position of all domain world's participants. A corresponding domain world AP (APdw) provides a semantic sharing of domain activities as well as of domain ontology.

Domain world activity (DW Activity) represented by APdw is aimed to the achievement of domain socio-economic, sociopolitical and socioproductive goals with cost minimization. APdw performance is achieved via Web portal.

\section{ACTIVITY PPROPOSITION (AP) BASED SELF- OGANIZATION}

Onto-surface constitutes the external level of human experience knowledge representation. Every Onto-surface's unit has a multisemantic position (AP-address) in the Activity Proposition net (AP-net) that represents set of triplets (APdwName, APName, OntoUnitName) and (APpw, APName, OntoUnitName). Recognition of MinContext in the current word's ambient determinates an actual word's AP-address.

On the other hand, every OntoUnitName relates to Web resource, containing its representation, namely, its meaning as well as modes of its keeping and its initialization. Note that AP represents the OntoUnit's utilization.

AP identification results from the text origins (see above) and from an analysis of Aexe's problems. Aexe problem indicates missing conditions for user activity support's continuation. In this case AmI-engine calls a dispatcher agent (Aforeman) that calls (depending on missing data type) one of AmI related APs. If it called Repair(name) agent, it considers the existing (an initial state) and required conditions (a goal state) as a target setting. A solution is a sequence of actions from initial state to a goal state. 
As AP's internal form is represented by a causal triple (I,C,E) (where I is an initial activity's state, $\mathrm{E}$ is a goal state (that is, an effect), and C (cause) is a description of I's transformation to E),

A solution is a path from initial to goal nodes. The search tree starts with just the root node, which represents the initial state.

Nodes that are generated physically form a queue structure, composed of APs, that is AP too.

If Repair(name) returns a missing data, Aexe weighs anchor.

\section{THE P2P/GRID}

"The term GRID refers to an emerging network based computing infrastructure providing security, resource access, information and other services that enable the controlled and coordinated sharing of resources among virtual organisations formed dynamically

by individuals and institutions with common interests " [14].

Since AP represents a scenario of an activity execution by means of activities and actions (realized by private applications, by software agents, by service providers and by other data handlers), it is a program of associated Web resource management. Thus THE Web involves AP based sharing of Web resources containing (and/or related to) the aforesaid tools. This implies an attaching of a corresponding computing infrastructure (THE GRID). Profession communities with common interests form THE GRID dynamically as well as individuals' and institutions' continuously supplement existing THE GRID by their AP nodes.

Obviously those THE GRID computers communicate directly with each other rather than via central THE servers (the opposite of client-server model) constituting THE P2P communication model.

An achievement of AmI owners' private goals is possible on basis of load-carrying contacts with a view a business, a scientific or/and cultural collaboration and transactions.

THE P2P computing is intended to complement existing client/server applications and to open up new opportunities to take advantage of the computing assets and resources of both individuals and organizations.

THE Grid/P2P should not be limited to the deployment of distributed computing on a known set of computers, of static topology and a stand-alone network of computer nodes. Furthermore it must take into account the dynamics of the computations. THE AmI nodes may join and leave the grid at any time. 


\section{CONCLUSION}

Just as a human behaviour is determinate by his knowledge, THE Web infrastructure unlike of other today's IT initiatives is produced from knowledge representation.

Majority of the software agent tools don't maintain the agents' development. Its objective is a providing the agents' interoperability in the networks or in the MASes. Software agents' development environments don't support a top-level total ontology creation.

THE Web initiative provides the knowledge engineering standard that grounds a functioning of split-level FIPA satisfying MAS (THE-mas). THEmas levels are

- Knowledge formative level (an experience representation, an ontology mapping, Web resource mapping, private resources mapping)

- An activity formative level (an administration of knowledge level's resources)

- Domain world formative level (an administration of domain related knowledge resources)

- Private world (i.e. a person's-, an enterprise's-, a government's. world) formative level (an administration of a private world related knowledge resources).

In that way constituted system will obtain as a personal intellectual assistant for maintenance of customer's profession/living activities, decision making and generating irrefragable answers. At that a communication's issues are solved due to the total ontology and unitized agents' organization.

Since Web interoperability is a function of knowledge representation, THE Web is an approach of Web interoperability achievement.

Activity Propositions and derived THE Ontology, THE GRID, THE P2P ground a novel approach of private Web platforms creation (Personal and Domain Worlds) as well as of these platforms interaction. As a result of PWs' interaction will be a dynamical generation of e-business, e-learning, emanagement, e-works and other Web services.

\section{REFERENCE}

1. M. Polanyi, Personal Knowledge, Harper \& Row, New York, 1958

2. ftp://ftp.cordis.lu/pub/ist/docs/istagscenarios2010.pdf;

3. Mika Klemettinen, User Centric \& Context AwareServices, AWSI Workshop, Tammsvik, Bro, Sweden,3.6.2003

4. Schank, Lebowitz, \& Birnbaum, Integrated Partial Parser 1980 
5. R. Schank and K. Colby, editors.Computer Models of Thought and Language. W. H. Freeman and Co., San Francisco, CA, 1973

6. R. C. Schank. Conceptual Information Processing. North-Holland, Amsterdam, 1975

7. R. Schank and R. Abelson. Scripts, Plans, Goals, and Understanding.Lawrence Erlbaum Associates, Hillsdale, NJ, 1977

8. R. Montague "English as a formal language", 1970

9.John F. Sowa, Concepts in the Lexicon, Problems and Issues, http://www.jfsowa.com/ontology/lexl.htm

10. The Columbia Encyclopedia, Sixth Edition. 2001

11. E-Slate: a software architectural style for end-user programming George Birbilis, Manolis Koutlis, Kriton Kyrimis, George Tsironis, George Vasiliou Computer Technology Institute Riga Feraiou 61Patras 262 21, Greece \{birbilis, koutlis, kyrimis, tsironis, vasiliou\}@cti.gr

12. M. Koutlis, P. Kourouniotis, K. Kyrimis, N. Renieri, "Inter-component communication as a vehicle towards end-user modeling", ICSE'98 Workshop on Component-Based Software Engineering, Kyoto, Japan, April 26-27, 1998.Available at < (http://www.sei.cmu.edu/ activities/cbs/icse98/papers/p7.html)>.

13. J. Roschelle, M. Koutlis, A. Reppening, et al.: "Developing Educational Software Components", IEEE Computer 10,9, special issue on Web based learning and collaboration, September 1999, pp. 50-58.

14. The Anatomy of the Grid: Enabling scalable virtual organizations, I. Foster, C. Kesselman and S. Tuecke, Int. J. Supercomputer Applic. 15, 3 (Fall 2001); see also www.globus.org/research/papers.html

15. Mario Bunge. Causality. The place of the causal principle in modern science. Cambridge: Harvard University Press 1959

16. W3C Working Group Note 11 February 2004. Web Services Architecture (http://www.w3.org/TR/2004/NOTE-ws-arch-20040211/)

17.FIPA Communicative Act Library Specification http://www.fipa.org/specs/fipa00037/XC00037H.htm 